\title{
Research on Hierarchical Algorithm of Wireless Sensor Network Based on Small World Network Model
}

\author{
Qichao Tang ${ }^{1, \text { a) }}$ \\ ${ }^{1}$ School of Chongqing University of Posts and Telecommunications, Chongqing 400065, China. \\ a) 2075721094@qq.com
}

Keywords: Hierarchical algorithm, Wireless sensor network, Small world network model

\begin{abstract}
The characteristics of the small world network is widely used in Wireless Sensor Networks. In this paper, Hierarchical Algorithm of Wireless Sensor Network Based on Small World Network Model (HASWNM) is proposed. Firstly,the algorithm divide the monitoring area into different sub-regions, and select the cluster node according to the data transmission reliability.Secondly, build shortcuts between cluster heads according to the model of DAS .Finally,we found that the WSNs had small average path length and large clustering coefficient, and lifecycle of network is obviously improved .
\end{abstract}

\section{INTRODUCTION}

In recent years, complex network has become one of the most important interdisciplinary research fields. As one of the characteristics of complex networks, the small world is widely used in realistic networks. The essential characteristics of the small world network are small average path length and large clustering coefficient. The study on the small world of complex network is introduced into the WSNs, has very important theoretical significance in analyzing the topology of WSNs, founding hidden rules and Predicting behavior of WSNs. For these reasons, scholars at home and abroad have provided a lot of methods to construct Wireless Sensor Networks having the characteristics of small world ${ }^{[1,2]}$. In this paper, Hierarchical Algorithm of Wireless Sensor Network Based on Small World Network Model (HASWNM) is proposed. We build a wireless sensor network with characteristics of small world by adding a certain proportion of super nodes and shortcut. Compared with algorithm of CSWN, TSWN and DASM, algorithm of HASWNM has obvious advantages in lifecycle of network and energy consumption.

\section{SYSTEM MODEL AND ALGORITHM FLOW}

\section{System Model}

a. N sensor nodes are randomly deployed in the square monitoring area of $\mathrm{M}^{*} \mathrm{M}$, and the sensor nodes are divided into ordinary nodes(L-sensor) and high performance nodes(H-sensor). Nodes are identified by ID, and the storage and computation energy is limited. Once the sensor node is deployed, it has been in a static state.

b. Storage, computing power of sink node is not limited, and sink nodes are fixed.

c. Assume that the communication radius of the node $i$ is $R_{c}(i)$ and the communication radius of the node $\mathrm{j}$ is $R_{c}(j)$.The necessary and sufficient condition for the direct communication between the node $\mathrm{i}$ and the node $\mathrm{j}$ is $R_{c}(i) \leq d_{i j} \Lambda R_{c}(j) \leq d_{i j}$, where $d_{i j}$ is the distance between the node $\mathrm{i}$ and the node $\mathrm{j}$.

\section{Algorithm Flow}

a. The sensor nodes in the monitoring area are divided into sub-regions, and the cluster nodes are elected.

In each sub-region, the sensor nodes form a single link. Selection of cluster head is based on the reliability of data transmission. Assume that there are $\mathrm{N}$ sensor nodes in a cluster, and a single link is formed according to the algorithm of PEGASIS. As is show in fig.1, if there are $\mathrm{m}$ sensor nodes on the left side of the cluster head node called $\mathrm{C}_{4}$, there are N-1-m sensor nodes on the right link of 
C4. As show in Fig.2, the entire cluster of data transmission reliability $R_{\text {cluster }}$ is expressed as ${ }^{[3]}$ :

$$
R_{\text {cluster }}=1-\left(1-R_{C H}\right)\left\{1-\left[1-\prod_{i=1}^{m}\left(1-R_{C M_{i}}\right)\right]\left[\left(1-\prod_{i=m+1}^{N-1-m}\left(1-R_{C M_{i}}\right)\right]\right\}\right.
$$

(1)

where $R_{C H}$ is the data reliability of the node of cluster head and $R_{C M_{i}}$ is data Reliability of common sensor nodes. The data reliability of node $\mathrm{i}$ is defined as $R_{\mathrm{CM}_{i}}=E_{r e}(\mathrm{i}) / E_{\text {init }}(\mathrm{i})$, where is the residual energy of node $i$ and $E_{\text {init }}(i)$ is the initial energy of node. The more the value of $R_{\text {cluster }}$ is, the greater possibility of becoming a cluster head.

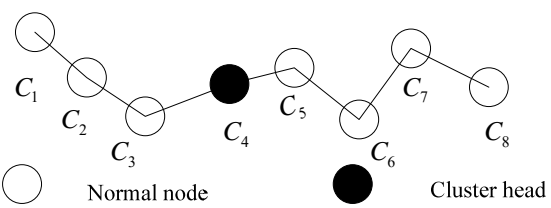

FIGURE1. Topology of sub-region

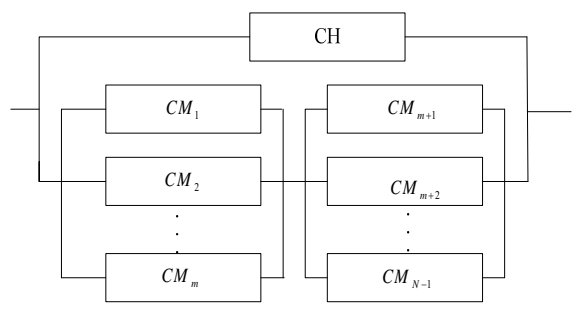

FIGURE2. Model of RBD

b. Determine the adaptive search area of the cluster head node $i$.

As is show in Fig.3, all nodes know that the sink node is located at $(0,0)$. Assume that straight line going through the node $\mathrm{i}$ and sink node is $l, y=m x+b$. The straight lines $l_{1}$ and $l_{2}$ going through the node $\mathrm{i}$ are symmetrical according to the straight line $l$ and $\theta$ is the angle between the straight lines $l_{1}$ and $l_{2}$, representing of the search area for node $i$. and the composition of the angle, the included angle of the region is the cluster head node $i$ search area ${ }^{[4]}$. The sensor nodes near the sink node should not only be responsible for the collection of the surrounding data, but also be responsible for a large number of data processing and forwarding data from the other nodes. This may cause energy consumption of the node is very fast. We can solve this problem by controlling the angle of the search area $\theta$. The smaller the distance between the node I and sink node, the bigger $\theta$ is $^{[5]}$. The node $\mathrm{i}$ has more choices when the value of $\theta$ becomes bigger, avoiding the phenomenon that some nodes have too high betweenness centrality ${ }^{[6]}$.

$$
\theta=\frac{d_{\max }-d_{i s}}{d_{\max }-d_{\min }} * \theta_{\text {init }}
$$

where $d_{\max }$ is the maximum distance between the cluster head node and the base station, $d_{\text {min }}$ is the minimum distance between the cluster head node and the base station, $d_{i s}$ is the distance between the current cluster head node and the base station and $\theta_{\text {init }}$ is the initial value of the searching angle $\theta$.

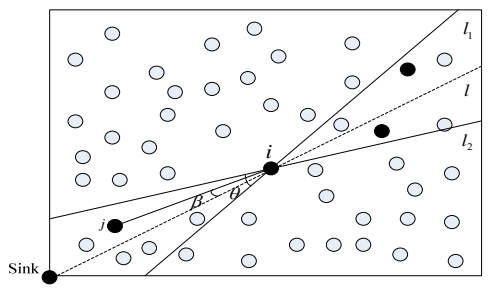

FIGURE3. Model of DAS

c. Determine candidate endpoint of shortcut ${ }^{[7,8]}$

Assume that the straight line going through the cluster head node $\mathrm{i}$ and node $\mathrm{j}$ in the communication range of node $\mathrm{i}$ is $l_{3}$ and $\beta$ is the angle between the straight lines $l_{3}$ and $l$. If node $\mathrm{j}$ is determined as the candidate endpoint of shortcut, it needs to be meet that $\beta<\theta / 2$.

If the cluster head $\mathrm{i}$ does not find the presence of the candidate endpoint of shortcut within the current adaptive search area, the cluster head will continue to expand the search range $\theta$ until the candidate endpoint of shortcut is found. If the cluster head i still does not find the candidate endpoint of shortcut when $\theta$ reaches to $360^{\circ}$, the ordinary nodes of the neighboring cluster are 
selected as the next hop node for data transmission.

d. Determine endpoint of shortcut

As the sensor nodes are deployed randomly, it will lead to the selection of cluster head is not necessarily the $\mathrm{H}$-sensor node. If the cluster head node $\mathrm{i}$ is L-sensor, the node $\mathrm{i}$ selects the node $\mathrm{j}$ closest to the head node $i$ as the next hop node of the node $i$. If the cluster head node $i$ is the Hsensor node, it will compare the current residual energy of node $i$ and the average residual energy of the cluster heads in the network. The average residual energy of the cluster heads $E_{\text {ave }}$ is described as

$$
E_{\text {ave }}=\frac{1}{m} \sum_{i=1}^{m} E_{\text {res }}(i)
$$

where $\mathrm{m}$ is the number of the cluster head, and $E_{\text {res }}(i)$ is the residual energy of node $\mathrm{i}$. If $E_{\text {res }}(i)>E_{\text {ave }}$, the cluster head node i selects the nearest node from the base station from the current candidate endpoint of shortcut as the next hop. If $E_{\text {res }}(i)<E_{\text {ave }}$, the candidate endpoints of shortcut can obtain a random number $\mathrm{Q}$ between 0 and 1.The first node that meets the condition $\mathrm{Q}<\mathrm{p}$ is selected as the next-hop node, where $\mathrm{p}$ is probability of reconnection.

\section{SIMULATION AND RESULTSANALYSIS}

TABLE1. Experimental Scene

\begin{tabular}{cc}
\hline Parameter & Value \\
\hline Range of the monitoring area & $\pi \times 1000 \times 1000 \mathrm{~m}^{2}$ \\
The number of nodes & 1000 \\
Initial angle & $10^{\circ}$ \\
Communication radius of common nodes $R_{L}$ & $100 \mathrm{~m}$ \\
Communication radius of nodes with high & $350 \mathrm{~m}$ \\
performance $R_{H}$ & $10 \mathrm{~J}$ \\
Initial energy of nodes with high performance & $1 \mathrm{~J}$ \\
Initial energy of common nodes & 0.5 \\
Probability of reconnection &
\end{tabular}

We define that lifecycle of network is the death time of the first node, $C(0)$ is clustering coefficient of network doesn't have the shortcut, and $C(H)$ is clustering coefficient of network has the shortcut. $L(H)$ is average path length of network has the shortcut, and $L(0)$ is average path length of network has the shortcut.

As show in Fig.4, we find that when number of nodes with high performance is between 40 to 90 , energy consumption of HASWNM is bigger than the other algorithm. HASWNM adopt the method of clustering and establish shortcuts between cluster heads. When the number of nodes with high performance is small, the network is easy to be not connected. With the increasing number of nodes with high performance beyond 90, energy consumption is smaller than the other algorithms. Because DASM search the shortcut within a fixed range, and TSWN search the shortcut within an adaptive range .But TSWN selects endpoint of shortcut randomly. From Fig.5, we find when the number of nodes with high performance is small, lifecycle of network of HASWNM isn't ideal because of high energy consumption. With the increasing number of nodes with high performance, lifecycle of network in HASWNM is better than the other algorithms. 


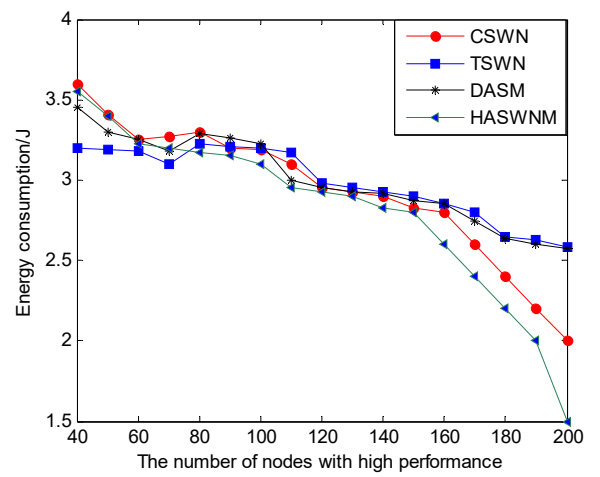

FIGURE4. The relationship between energy consumption and number of nodes with high performance

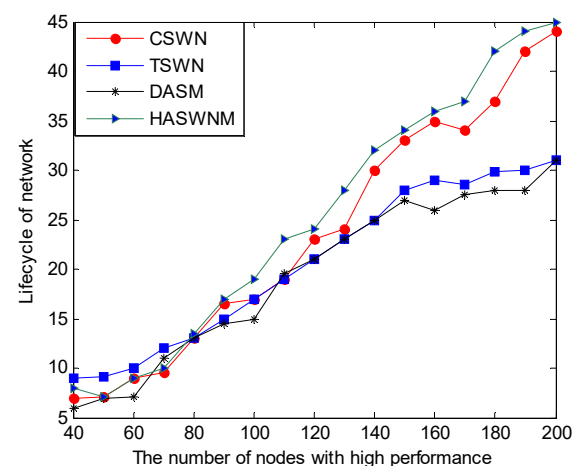

FIGURE5. The relationship between lifecycle of network and number of nodes with high performance

The characteristic of small world can be seen in wireless network according to $L(H) / L(0)$ and $C(H) / C(0)$ in Fig.6.With the increasing number of nodes with high performance, average path length of network is becoming smaller. When the number of nodes with high performance reaches 100 , average path length of network is nearly unchanged. At the same time, the network has the high clustering coefficient. We can also find that optimal number of clusters in this network is 100 .

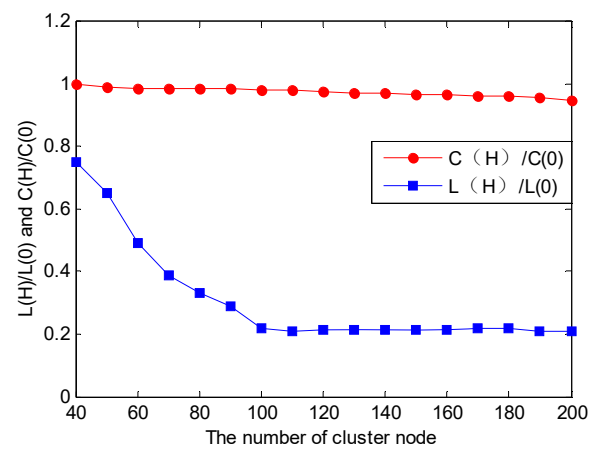

FIGURE6. The characteristic of small world in HASWNM

\section{CONCLUSION}

The characteristic of small world in wireless network adopting algorithm of HASWNM can be found. Algorithm

of HASWNM has some advantages. Firstly, selection of cluster head is according to the reliability of data transmission. Secondly, when cluster head selects the next-hop node of shortcut, it consider own energy because of the energy consumption focusing on the data transmission phase. Only If $E_{\text {res }}(i)>E_{\text {ave }}$, the cluster head node $\mathrm{i}$ selects the nearest node from the base station from the current candidate endpoint of shortcut as the next-hop node. Thirdly, the searching area of cluster node is variable according to distance between the node and base station. By simulation, it proved that algorithm of HASWNM have advantage in energy consumption and lifecycle of network. 


\section{ACKNOWLEDGMENTS}

This work was financially supported by the Program for Changjiang Scholars and Innovative Research Team in University (IRT1299), Project of CSTC (CSTC2012jjA40044, CSTC2013yykfA40010) and special fund of Chongqing key laboratory (CSTC) fund.

\section{REFERENCES}

[1]. Guidoni D L, Mini R A F, Loureiro A A F. On the design of resilient heterogeneous wireless sensor networks based on small world concepts[J]. Computer Networks, 2010, 54(8):1266-1281.

[2]. Sooktip T, Wattanapongsakorn N, Coit D W. System reliability optimization with k-out-of-n subsystems and changing $\mathrm{k}[\mathrm{C}] / /$ International Conference on Reliability, Maintainability and Safety. IEEE, 2011:1382-1387.

[3].Z D Zhou, P Hu, F M Li. Reliable scheme for the cluster-based communication protocol in wireless sensor networks [J]. Journal on Communications, 2008, 29(5):114-121.

[4]. Guidoni D L, Mini R A F, Loureiro A A F. On the design of heterogeneous sensor networks based on small world concepts[C]// International Symposium on Modeling Analysis and Simulation of Wireless and Mobile Systems, MSWIM 2008, Vancouver, British Columbia, Canada, October. DBLP, 2008:309-314.

[5].Q H Xie, J Huang. Construction of small world characteristics in wireless sensor networks based on dynamic search region [J]. Journal of Southeast University, 2012, 42 (4): 593-598.

[6]. Asif W, Qureshi H K, Rajarajan M. Variable rate adaptive modulation (VRAM) for introducing small-world model into WSNs[C]// Information Sciences and Systems. 2013:1-6.

[7].Y D Hu. Research on topology control in heterogeneous wireless sensor networks based on small world [D]. Jiangsu University, 2016.

[8].X C Li. Research on Routing Algorithm for wireless sensor networks based on small world model [D]. Nanjing University of Posts and Telecommunications, 2012. 